\title{
Dielectric Behavior of Binary Mixtures of 2,3-Dichloroaniline with 2-Methoxyethanol Using Microwave X-Band
}

\author{
BHUPESH NEMMANIWAR and POTHAJI KADAM*
}

Physics Research Laboratory, Department of Physics, Yeshwant Mahavidyalaya, Nanded - 431605(MS), India

pothajikadam@yahoo.com

Received 15 February 2014 / Accepted 15 March 2014

\begin{abstract}
Densities, viscosities, refractive index, dielectric constant $\left(\varepsilon^{\prime}\right)$ and dielectric loss $\left(\varepsilon^{\prime \prime}\right)$ of 2,3-dichloroaniline (2,3-DCA) + 2-methoxyethanol (2-ME) for different mole fractions of 2,3-dichloroaniline in binary mixture have been measured at single microwave frequency 10.985 $\mathrm{GHz}$ at $40{ }^{\circ} \mathrm{C}$ by Surbers technique at microwave X-band. The values of dielectric parameters $\left(\varepsilon^{\prime}\right)$ have been used to evaluate the molar polarization $\left(\mathrm{P}_{12}\right)$, loss tangent $(\tan \delta)$, excess viscosities $(\Delta \eta)$ and excess dielectric constant $\left(\Delta \varepsilon^{\prime}\right)$ have also been estimated. These parameters have been used to explain the formation of complexes in the system. It is found that dielectric constant $\left(\varepsilon^{\prime}\right)$, dielectric loss $(\varepsilon ")$, loss tangent $(\tan \delta)$, molar polarization $\left(\mathrm{P}_{12}\right)$ varies non-linearly but activation energy $(\mathrm{Ea})$, viscosity $(\eta)$, density $(\rho)$ and refractive index $(n)$ varies linearly with increasing mole fraction in binary mixture of 2,3-dichloroaniline (2,3-DCA) + 2-methoxyethanol (2-ME). Hence, solute-solvent molecular associations have been reported.
\end{abstract}

Keywords: Molecular interaction of polar liquids, 2,3-Diachloroaniline (2,3-DAC), 2- Methoxyethanol (2-ME), Binary mixtures, Excess parameters

\section{Introduction}

Recently several workers ${ }^{1-5}$ have reported the utility of dielectric measurement while studying the molecular interaction in a binary mixture of liquids. Rana and $\mathrm{Vyas}^{6}$ have studied the relaxation time and distributed parameters for binary mixture of 3-bromoanline and 1-proponal and suggested the interaction between the constitute molecules. Das and Hazra $^{7}$ have studied the density, viscosity and excess parameters of binary mixture of 2-ethoxyethanol (2-EE) with $N, N$-dimethylacetamide (DMA) and explained the deviation of excess parameters in terms of interaction between unlike molecules in the binary mixture. Gupta et al., ${ }^{8}$ have studied the molar polarization and free energy activation of cyclohexylamide (CHA) and throw a light on the dynamic characteristics of molecular association in the binary mixture.

In this paper our aim is to provide the information regarding the possibilities of complex formation due to molecular association between two polar liquids namely 2,3-DCA and 
2-ME at $40{ }^{0} \mathrm{C}$. In this paper the dielectric properties and molecular behavior of 2,3-dichloroaniline (2,3-DCA) with 2-methoxyethanol (2-ME) and their binary mixture are discussed. We report here the values of dielectric constant $\left(\varepsilon^{\prime}\right)$ and dielectric loss $\left(\varepsilon^{\prime \prime}\right)$ obtained experimentally by employing Surber's technique of measuring reflection coefficient from air dielectric boundary of the liquid.

\section{Experimental}

2,3-Dichloroaniline (GC Grade) from Sigma-Aldrich, Germany and 2-methoxyethanol (AR Grade) were obtained from SD Fine-Chem Limited, Mumbai (India). Without further purification the two liquids according to their proportions by volume were mixed well and kept 6 hours in well stoppered bottles to ensure good thermal equilibrium. 2,3-DCA was used as solute and 2-ME as solvent.

\section{Measurements}

The dielectric constant $\left(\varepsilon^{\prime}\right)$ and dielectric loss $\left(\varepsilon^{\prime \prime}\right)$ have been measured using microwave $\mathrm{X}$-band bench oscillating frequency of $10.985 \mathrm{GHz}$ at $40{ }^{\circ} \mathrm{C}$ using source of Reflex klystron 2 K 25 (USSR) by Surber ${ }^{9}$. The densities and viscosities of the pure components and their mixtures were measured by using DMA 35 portable vibrating density meter. Anton paar Autria (Europe) having accuracy of density $0.001 \mathrm{~g} / \mathrm{cm}^{3}$, repeatability $0.0005 \mathrm{~g} / \mathrm{cm}^{3}$ and resolution $0.0001 \mathrm{~g} / \mathrm{cm}^{310}$ and viscosity by LVDL, V-pro II Brook field viscometer (USA) ${ }^{11}$.

A plunger waveguide is converted into a cavity by introducing a coupling hole in the entrance and shorting the other end with the calibrated plunger. The sample occupies the entire volume of the cavity. The frequency was kept constant and the length of the plunger cavity was changed. Hence several nodes appear as one increase the length of the cavity plunger. Whenever the length of the cavity equals the half integral multiples of the waveguide wavelength inside the medium, the plunger waveguide resonates. The distance through which the plunger is move between two successive cavity nodes gives half of the wavelength $\left(\lambda_{d}\right)$ of the microwave inside the medium. The measurement of reflected power at resonance gives the attenuation coefficient of the sample ${ }^{12}$.

Refractive indices for sodium D-line were measured by using Abbe's refractometer, having accuracy up to the third place of decimal microwave power measured by PM-437 (Attest) power meter, Chennai, India. Rectangular wave guide working $\mathrm{TE}_{10}$ mode, $10 \mathrm{~dB}$, Vidyut Yantra Udyog, India. To hold the liquid sample in the liquid cell, thin mica window whose VSWR and attenuation were neglected is introduced between the cell and rest of microwave bench. The values of $\left(\varepsilon^{\prime}\right)$ and $\left(\varepsilon^{\prime \prime}\right)$ for low loss liquids are calculated according to Singh et al., ${ }^{12}$ formula.

$$
\begin{aligned}
\varepsilon^{\prime} & =\left(\frac{\lambda_{0}}{\lambda_{c}}\right)^{2}+\left(\frac{\lambda_{0}}{\lambda_{d}}\right)^{2} \\
\varepsilon^{\prime \prime} & =\frac{2}{\pi}\left(\frac{\lambda_{g}}{\lambda_{d}}\right)\left(\frac{\lambda_{0}}{\lambda_{d}}\right)^{2}\left(\frac{d \rho}{d n}\right)
\end{aligned}
$$

Where $\lambda_{\mathrm{c}}$ is the cut-off wavelength, $\lambda_{0}$ is the free space wavelength, $\lambda_{\mathrm{d}}$ is the wavelength in dielectric medium, $\lambda_{\mathrm{g}}$ is the wavelength in empty wave guide parameters and $\rho$ is the inverse voltage standing wave ratio.

The values of molar polarization $\left(\mathrm{P}_{12}\right)$ of the binary mixture were obtained by using the formula ${ }^{12-13}$. 


$$
P_{12}=\left[\frac{\left(\epsilon^{\prime}-1\right)}{\left(\epsilon^{\prime}+2\right)}\right]\left[\frac{M_{1} X_{1}+M_{2} X_{2}}{\rho}\right]
$$

Where $M_{1}$ and $M_{2}$ molecular weights of solute and solvent $X_{1}$ and $X_{2}$ are the mole fraction of solute and solvent and $\rho$ is the density of binary mixture.

\section{Results and Discussion}

The values of density $(\rho)$, viscosity $(\eta)$, refractive index $(n)$, dielectric permittivity $\left(\varepsilon^{\prime}\right)$, dielectric loss $\left(\varepsilon^{\prime \prime}\right)$, loss tangent $(\tan \delta)$, activation energy $(\mathrm{Ea})$ and molar polarization $\left(P_{12}\right)$ for viscous flow with increasing mole fraction $(\mathrm{X})$ of 2,3-DCA for the binary mixtures of 2,3-DCA+2-ME are reported in Table 1.

Table 1. Mole fraction (X) of 2,3-DCA density $(\rho)$, viscosity $(\eta)$, refractive index $(n)$, dielectric constant $\left(\varepsilon^{\prime}\right)$, dielectric loss $\left(\varepsilon^{\prime \prime}\right)$, loss tangent $(\tan \delta)$, activation energy $\left(E_{a}\right)$ and molar polarization $\left(P_{12}\right)$ for binary liquid mixture at $40{ }^{\circ} \mathrm{C}$

\begin{tabular}{ccccccccc}
\hline $\mathrm{X}$ & $\begin{array}{c}\rho \\
\mathrm{g} / \mathrm{cm}^{3}\end{array}$ & $\eta \mathrm{cp}$ & $\mathrm{n}$ & $\varepsilon^{\prime}$ & $\varepsilon^{\prime \prime}$ & $\tan \delta$ & $\begin{array}{c}\mathrm{E}_{\mathrm{a}}, \\
\mathrm{kcal} / \mathrm{mol}\end{array}$ & $\mathrm{P}_{12}$ \\
\hline 0.0000 & 0.9566 & 1.25 & 1.393 & 5.3417 & 0.4279 & 0.08011 & 6.5443 & 47.0454 \\
0.08697 & 1.0170 & 1.48 & 1.430 & 6.8107 & 05371 & 0.0788 & 6.5494 & 54.1954 \\
0.18185 & 1.0694 & 1.74 & 1.444 & 7.1217 & 0.5463 & 0.07671 & 6.6149 & 57.5629 \\
0.28576 & 1.1724 & 2.04 & 1.466 & 8.8458 & 0.6407 & 0.07243 & 6.8490 & 59.2929 \\
0.40006 & 1.2236 & 2.33 & 1.496 & 9.3269 & 0.6385 & 0.06846 & 6.9317 & 69.271 \\
0.52637 & 1.2280 & 2.63 & 1.511 & 10.2342 & 0.7401 & 0.07232 & 7.0071 & 74.8408 \\
0.66672 & 1.2750 & 2.96 & 1.531 & 11.6050 & 07929 & 0.06832 & 7.0806 & 81.5468 \\
0.82356 & 1.3231 & 3.25 & 1.548 & 12.7589 & 0.8124 & 0.06367 & 7.1388 & 88.4352 \\
1.0000 & 1.3784 & 3.74 & 1.580 & 13.4267 & 0.9651 & 0.07188 & 7.2261 & 87.0645 \\
\hline
\end{tabular}

The density of binary mixture of 2,3-DCA+2-ME are increasing as mole fraction of 2,3-DCA in the binary mixture is increasing. This is expected because density of pure 2,3 -DCA is more than that of pure 2-ME. It can also be seen that viscosity $(\eta)$, refractive index (n) and activation energy (Ea) is increasing with increase in mole fraction of 2, 3-DCA in the binary mixture. This increase in $\eta, \mathrm{n}$ and $\mathrm{Ea}$ is further supported by the increase in density of the binary mixture with increase in mole fraction of 2, 3-DCA.

Increases in viscosity $(\eta)$ for the acid amine and 2-ME mixture due to the formation of dissociated ions in the mixture. Which is exothermic and depends upon the acidic strength of 2-ME. Since in the present paper the maximum viscosity for 2,3-DCA may be associated with the formation of dissociated ions in the mixture and due to the more acidic character of 2,3-DCA. The spectacular increases in viscosity $(\eta)$ may also be attributed to the mutual viscosity of the alcohols and amine molecules.

Viscosity $(\eta)$ increases as mole fraction of 2, 3-DCA in the binary mixture increases. Increase in viscosity of binary mixture can be attributed to increase in effect of hydrogen bonding with decrease in mole fraction of 2-ME. This is because pure 2,3-DCA is more viscous than pure 2-ME.It is due to presence of strong hydrogen bonding in 2,3-DCA, more number of carbon atoms and long straight chain compound.

The variation of molar polarization $\left(\mathrm{P}_{12}\right)$ versus mole fraction of 2,3-DCA as shown in Figure 1. It shows that the increases in $\mathrm{P}_{12}$ with increase in mole fraction of 2,3-DCA in binary mixture also indicates the increases in the effect of hydrogen bonding in the binary mixture. 
Intersection of straight line at $\mathrm{X}=0.48$ mole fraction of 2,3-DCA representing separate region of high and low concentration of 2,3-DCA can be interpreted as the point of maximum concentration the complex which corresponds to 1:1 complex for the system. Similar results have been reported by Nimkar ${ }^{14}$.

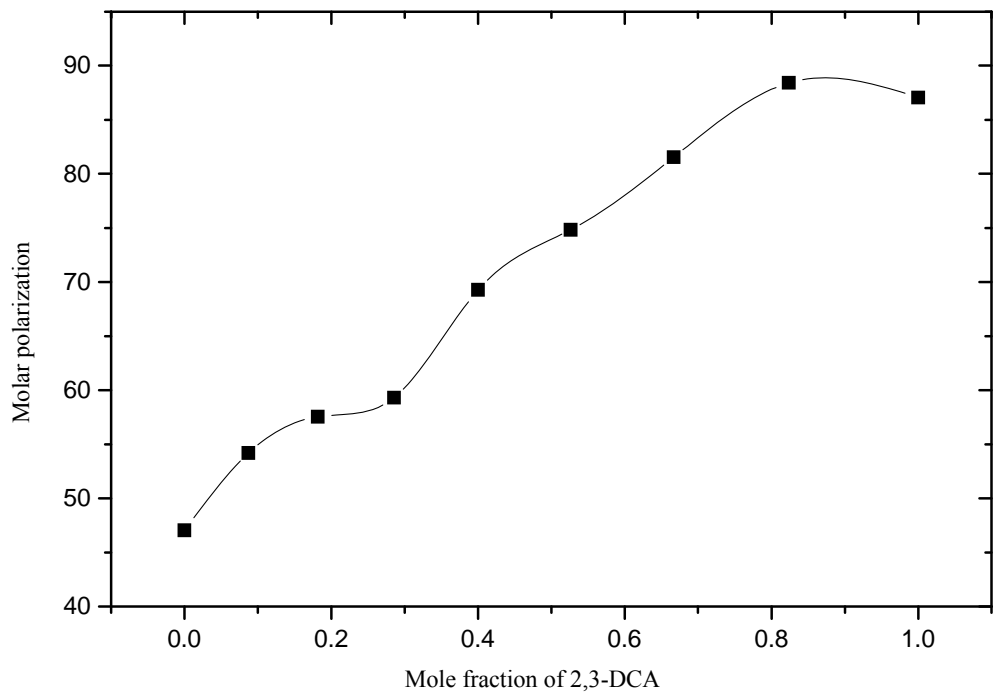

Figure 1. Plot of molar polarization $\left(\mathrm{P}_{12}\right)$ with mole fraction of 2,3-DCA+2-ME

The excess parameters are calculated by the formula defined by Singh and Sharma ${ }^{12}$.

$$
\Delta Y=Y_{m}-\left(X_{1} Y_{1}+X_{2} Y_{2}\right)
$$

Where $Y_{m^{-}}$is dielectric parameters for the mixture, $Y_{1}$ and $Y_{2}$ are the values for pure components, $X_{1}$ and $X_{2}$ are their respective mole fraction. For ideal mixture, the excess parameters are zero, but real mixtures have finite values. The excess may be positive or negative depending on complex formation.

Excess viscosity increases with mole fraction of 2,3-DAC in the binary mixture and it is maximum at $\mathrm{X}=0.4$ mole fraction of $2,3-\mathrm{DAC}$ and it is decereses with decreses in mole fraction of 2-ME as shown in Figure 2. Decereses in viscosity of binary mixture can be attributed to decereses in the effect of hydrogen bonding with increses in mole fraction of 2,3-DAC. This is because 2,3-DAC is more viscous than 2-ME. It is due to presences of strong hydrogen bonding in 2,3-DAC and more number of carban atoms and long chain straight chain compound.

The excess dielectric constant $\left(\Delta \varepsilon^{\prime}\right)$ of the mixture was plotted against the mole fraction of 2,3-DCA in the mixture at $40{ }^{\circ} \mathrm{C}$ is shown in Figure 3. From figure it can be seen that the variation of excess dielectric constant $\left(\Delta \varepsilon^{\prime}\right)$ versus mole fraction $(\mathrm{X})$ of 2,3-DCA as shown in Figure 3. From graph $\Delta \varepsilon^{\prime}$ is positive over entire concentration rang of mole fraction of 2,3-DCA. Investigation on concentration dependent excess dielectric constant $\left(\Delta \varepsilon^{\prime}\right)$ values of mixed dipolar solvents provides information regarding change in number of parallel alignment dipoles contributed to the dielectric polarization. The strength of hydrogen bonded interactions and the molar ratio of stable adduct. The similar results have been reported by Kinart et al., ${ }^{15}$ and Nemmaniwar ${ }^{16}$. The maximum peak of excess dielectric constant $\left(\Delta \varepsilon^{\prime}\right)$ at $X=0.2857$ mole fraction of 2,3-DCA. 


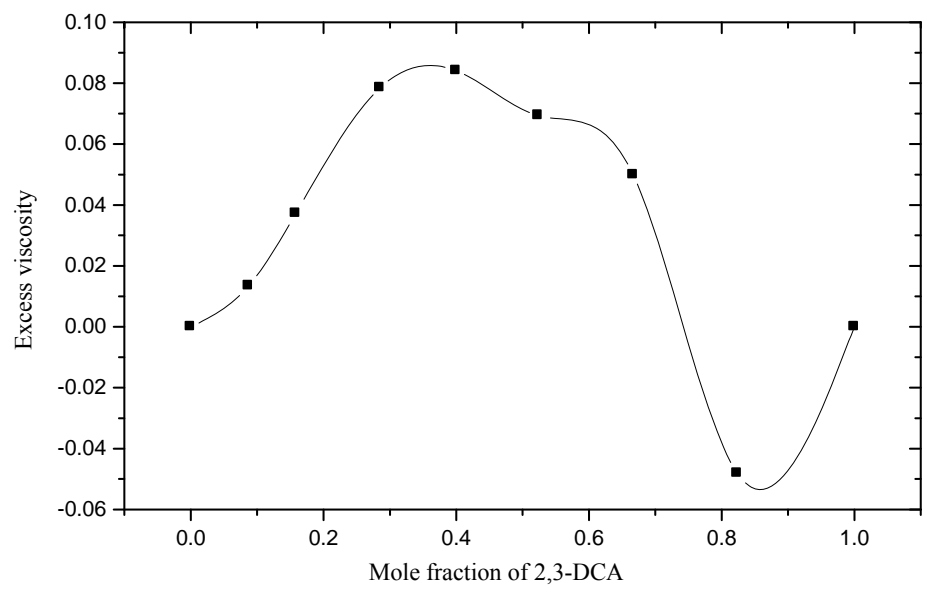

Figure 2. Plot of excess viscosity $(\Delta \eta)$ with mole fraction of 2,3-DCA+2-ME

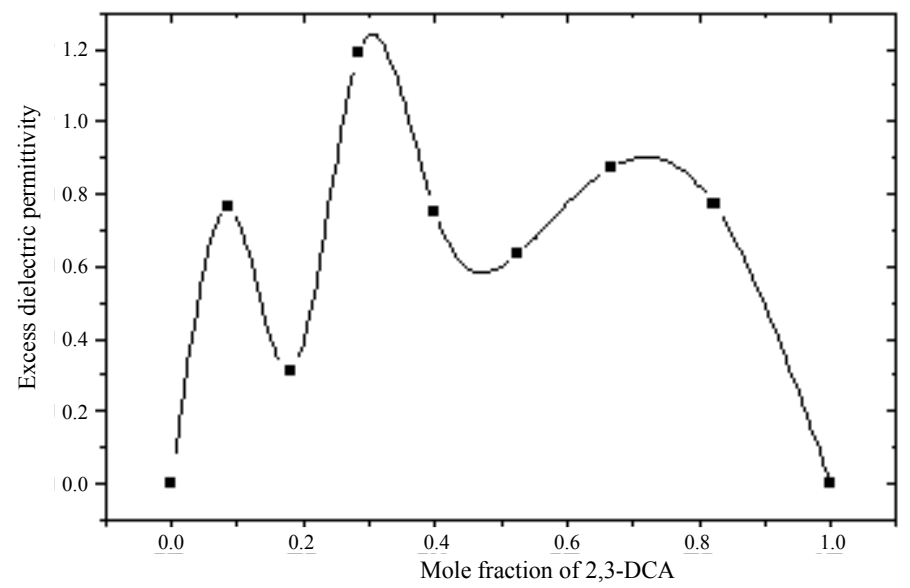

Figure 3. Plot of excess dielectric constant $\left(\Delta \varepsilon^{\prime}\right)$ with mole fraction of 2,3-DCA+2-ME

\section{Conclusion}

The dielectric constant, dielectric loss, loss tangent, viscosity, density, refractive index and excess parameters have been reported for binary mixture 2,3-DCA+2-ME at the various concentrations. These suggest the strong interaction between the alcohols and amine molecules. Molar polarization $\left(\mathrm{P}_{12}\right)$ curve suggests 1:1 type complex formation in the binary mixture. The excess viscosity $(\Delta \eta)$ values are positive for the whole composition range for 2, 3-DCA. This is due to charge transfer and hydrogen bonding interactions which leads to the formation of complex species between unlike molecules thereby resulting in positive excess viscosity values. As concentration 2, 3-DCA decreases, the maxima position also decreases. As the molecular size increases, the magnitude of excess viscosity increases i.e. becomes more positive showing a tendency to the ideal behavior.

The positive values of $\Delta \varepsilon^{\prime}$ indicates that molecules of the mixture form multimer structure via hydrogen bonding in such a way that the effective dipole moments are increased. 


\section{Acknowledgement}

The authors are thankful to the Principal, Yeshwant Mahavidyalaya Nanded for providing necessary laboratory facilities.

\section{References}

1. $\quad$ Sengwa R J, Indian J Pure Appl Phys., 2003, 41, 295-300.

2. Gupta K K, Bansal A K, Sing P J and Shrama K S, Indian J Pure Appl Phys., 2004, 42, 849-853.

3. Oster G, J Am Chem Soc., 1946, 68(10), 2036-2041; DOI:10.1021/ja01214a050

4. Kamble S, Sudake Y S and Mehrotra S C, J Koren Chem Soc., 2011, 55(3), 373.

5. Rana V A and Vyas A D, Indian J Pure Appl Phys., 2001, 39(5), 316-320.

6. $\quad$ Rana V A and Vyas A D, Indian J Phys., 2004, 78B, 165.

7. Das D and Hazra D K,Indian J Phys., 2003,77,519.

8. Gupta K K, Bansal A K, Singh P J and Sharma K S, Indian J Pure Appl Phys., 2003, 41, 801-805.

9. $\quad$ Surber W H, Appl J Phys., 1948, 19, 514.

10. Nemmaniwar B, Mokle S and Kadam P L, Pak J Chem., 2013, 3(2), 1.

11. Hill N E, Vaughan W E, Price A H and Davies M, Dielectric properties and Molecular Behavior; Van Nostrand: London, 1966.

12. Vural U S, Muradoglu V V and Vural S, Bull Chem Soc Ethiop., 2011, 25, 111-118.

13. Maharolkar A P, Sudkes Y, Kamble S, Tidar N D, Murugkar A G, Patil S S, Khirade P W and Mehrotra S C, Int J Chem., 2010, 2, 250.

14. Kinart C M and Klimczak M, J Mol Liq., 2009, 148(2-3), 132-139; DOI:10.1016/j.molliq.2009.07.009

15. Sengwa R J and Khatri V, Thermochimica Acta, 2010, 506(1-2), 47-51; DOI:10.1016/j.tca.2010.04.013

16. Nemmaniwar B G, .Kalyakar N V and Kadam P L, Orbital Elec J Chem., 2013, 5(1), 1-6. 